\title{
Endopeptidase Asp-N in Ammonium Bicarbonate Protein Cleavage Reagent
}

National Cancer Institute

\section{Source}

National Cancer Institute. Endopeptidase Asp-N in Ammonium Bicarbonate Protein

Cleavage Reagent. NCI Thesaurus. Code C161882.

A protein cleavage reagent comprised of a form of the bacterial endopeptidase Asp- $\mathrm{N}$ in a solution containing ammonium bicarbonate that cleaves proteins into peptides at the $\mathrm{N}$-terminal side of an aspartate or glutamate residue. 